\title{
Quantum dot state initialization by control of tunneling rates
}

\author{
Tobias Wenz, ${ }^{1}$ Jevgeny Klochan, ${ }^{2}$ Frank Hohls, ${ }^{1, *}$ Thomas Gerster, ${ }^{1}$ Vyacheslavs Kashcheyevs, ${ }^{2, \dagger}$ and Hans W. Schumacher ${ }^{1}$ \\ ${ }^{1}$ Physikalisch-Technische Bundesanstalt (PTB), Bundesallee 100, 38116 Braunschweig, Germany \\ ${ }^{2}$ Department of Physics, University of Latvia, Jelgavas Street 3, LV 1004 Riga, Latvia
}

(Received 6 March 2019; published 20 May 2019)

\begin{abstract}
We study the loading of electrons into a quantum dot with dynamically controlled tunnel barriers. We introduce a method to measure tunneling rates for individual discrete states and to identify their relaxation paths. Exponential selectivity of the tunnel coupling enables loading into specific quantum dot states by tuning independently energy and rates. While for the single-electron case orbital relaxation leads to fast transition into the ground state, for electron pairs triplet-to-singlet relaxation is suppressed by long spin-flip times. This enables the fast gate-controlled initialization of either a singlet or a triplet electron pair state in a quantum dot with broad potential applications in quantum technologies.
\end{abstract}

DOI: 10.1103/PhysRevB.99.201409

The ability to initialize a discrete quantum state by coupling to a cold external environment is an essential resource for many quantum technologies, providing, for example, a template to encode quantum information, an initial pure state for quantum sensing, or a supply of ancillary qubits for quantum error correction $[1,2]$. In solid-state quantum platforms such as semiconductor quantum dots (QDs), initialization strategies vary from simple cooling by spontaneous relaxation $[1,3]$ to control of tunneling to adjacent electron reservoir(s) via energy alignment [4] or Pauli blockade [5]. However, options to tune the initialization rate or the targeted quantum state (e.g., selecting between a singlet or a triplet) are limited with these energy-based approaches. On the other hand, exponential tunability of QD tunneling rates, which has recently been shown to be highly effective [6] for optimizing the speed-precision trade-off of single-electron (SE) pumps in quantum metrology [7], has not yet been exploited to combine the speed and the selectivity in initializing individual orbital and/or spin states of electrons confined to QDs. Hence, developing a strategy to control quantum-state-specific tunneling into and out of a QD could open new ways for the efficient initialization of qubits and enable fast on-demand sources of specific few-particle quantum states for electron quantum optics [8-10] or the transfer of quantum information between static qubits [11-14].

In this work, we perform spectroscopy of quantum-state energies and tunnel couplings for a dynamic QD operated as a SE pump by loading and capturing electrons near the Fermi edge. When the lowest QD levels are close to the Fermi energy $E_{\mathrm{F}}$, incomplete loading of electrons can occur $[7,15]$

\footnotetext{
*frank.hohls@ptb.de

†slava@latnet.lv
}

Published by the American Physical Society under the terms of the Creative Commons Attribution 4.0 International license. Further distribution of this work must maintain attribution to the author(s) and the published article's title, journal citation, and DOI. and the capture probability is determined by a competition of tunnel coupling, energy level positions [16], and loading times. We describe the experimental data by a simple model that enables quantitative spectroscopy for the rates of intunneling into the QD. Measurements reveal that electrons can tunnel either directly or via excited orbital states into the SE ground state. In the latter case SE capture is mediated by fast orbital relaxation. In contrast, relaxation for electron pair states requiring a singlet-triplet spin transition occurs on a much longer timescale than the loading times. In this regime of electron pair capturing, our data clearly indicate loading into specific singlet or triplet states as a function of gate voltages, thereby enabling gate-controlled fast quantum-state initialization of QDs.

Measurements were performed on a QD based on a $\mathrm{GaAs} / \mathrm{AlGaAs}$ heterostructure as typically used for tunable barrier SE pumps [6,17] [Fig. 1(a)]. From the twodimensional (2D) electron system, a channel was formed by shallow wet etching and two Ti/Au finger-shaped Schottky gates were deposited on top. At low temperatures $\left(T_{\text {bath }} \approx\right.$ $100 \mathrm{mK})$, a QD is formed between the entry and exit gates by applying negative voltages $V_{\text {entry }}$ and $V_{\text {exit }}$. Biased cooldown with $+70 \mathrm{mV}$ applied to both gates was used to increase device stability [18].

The state of the QD is controlled by a time-dependent signal $V_{\text {entry }}(t)$ with repetition frequency $f=1 / T$ applied to the entry gate, while $V_{\text {exit }}$ is kept constant. The shape of the clock signal [Fig. 1(b)] is designed to drive the QD through three distinct phases [Fig. 1(c)]: (i) For a time $T_{\text {load }}$, a number of lowest-energy states become available for electrons to tunnel onto an initially empty QD. (ii) The potential energy of the QD is raised, and some electrons may escape back to the source as the entrance tunnel barrier is gradually pinched off. This allows separation of the electron states based on the difference in the backtunneling rates [19]. (iii) All captured electrons are emitted to the drain through the exit barrier. The output current, $I=\langle n\rangle e f$, measures the average of the number $n$ of electrons captured by the end of phase (ii). 

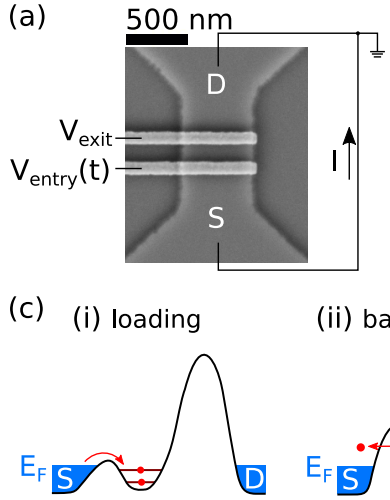

(c) (i) loading (b)

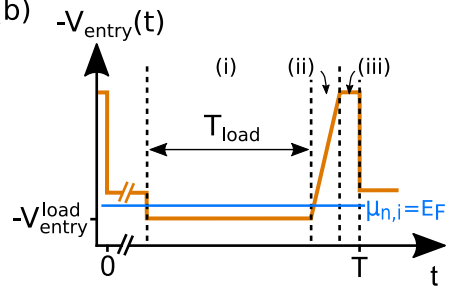

(ii) backtunneling

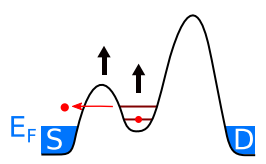

(iii) ejection

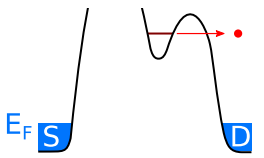

FIG. 1. (a) Scanning electron micrograph of a SE pump. (b) A wave form with period $T=1 / f$ is applied to the entry gate which moves the energy levels $\mu_{n, i} \propto-V_{\text {entry }}(t)+$ const. Loading of the QD is possible while $\mu_{n, i}<E_{\mathrm{F}}$. (c) Measurement cycle: (i) Electrons are loaded. (ii) Backtunneling of higher energy electrons. (iii) Captured electrons are ejected to drain.

A large difference in energies and tunnel couplings between one- and two-electron states is the basis for accurate tuning of the number of remaining electrons for quantum metrology of electrical current [7]. In the example shown in Fig. 2(a), the voltage $V_{\text {exit }}$ is used to control the backtunneling rates and hence the outcome of phase (ii). Three plateaus of quantized current corresponding to $\langle n\rangle=1,2,3$ are measured. Adding $V_{\text {entry }}^{\text {load }}$ to the tuning parameters reveals a 2D map of $\langle n\rangle$ as a function of $V_{\text {entry }}^{\text {load }}$ and $V_{\text {exit }}$, shown in Fig. 2(b). $V_{\text {entry }}^{\text {load }}$ is the extreme value of $V_{\text {entry }}(t)$, held constant for a time $T_{\text {load }}$ during the loading phase (i) [Fig. 1(b)].

We first consider the series of antidiagonal steps highlighted by three blue lines in Fig. 2(b). We identify these lines as resonances of the source Fermi energy $E_{\mathrm{F}}$ and the energies $\mu_{n, i}$ of distinct quantum states $i=0,1,2 \ldots$ available for the first $(n=1)$ electron. If $\mu_{1, i}<E_{\mathrm{F}}$ during the loading phase, the state $i$ contributes to initialization with an intunneling rate $\gamma_{1, i}$. The values of both $\mu_{n, i}$ and $\gamma_{n, i}$ are tuned by the gate voltages, leading to the qualitatively different initialization conditions sketched in Fig. 2(c). In configuration (1), only the ground state $\mu_{1,0}$ is available for loading, whereas the excited state $\mu_{1,1}>E_{\mathrm{F}}$ is energetically forbidden. Here, an electron can only be loaded if $\gamma_{1,0}$ is sufficiently large compared to $1 / T_{\text {load. }}$. The corresponding values of control voltages $\left(V_{\text {entry }}^{\text {load }}\right.$, $\left.V_{\text {exit }}\right)$ are marked (1) in Fig. 2(b). Configuration (2) is energetically similar to configuration (1), but the entry barrier is higher [see Fig. 2(c)], and thus the intunneling rate of the only energetically available state is much lower, $\gamma_{1,0} \ll 1 / T_{\text {load }}$. Hence loading at (2) fails (negligible $\langle n\rangle$ ).

In configuration (3) a second level is energetically available and can contribute to loading. This higher energy state $i=1$ is separated from the source by an effectively lower tunnel barrier resulting in an exponentially stronger coupling compared to the ground state $i=0$. In this regime $\gamma_{1,0} \ll 1 / T_{\text {load }}<\gamma_{1,1}$, and the loading takes place predominantly via state $i=1$.

We develop the above qualitative picture into a quantitative model for tunneling rate spectroscopy. A sufficiently sharp Fermi edge ensures that the processes of tunneling in (i) and out (ii) of the dot [see Fig. 1(b)] are temporally separated.

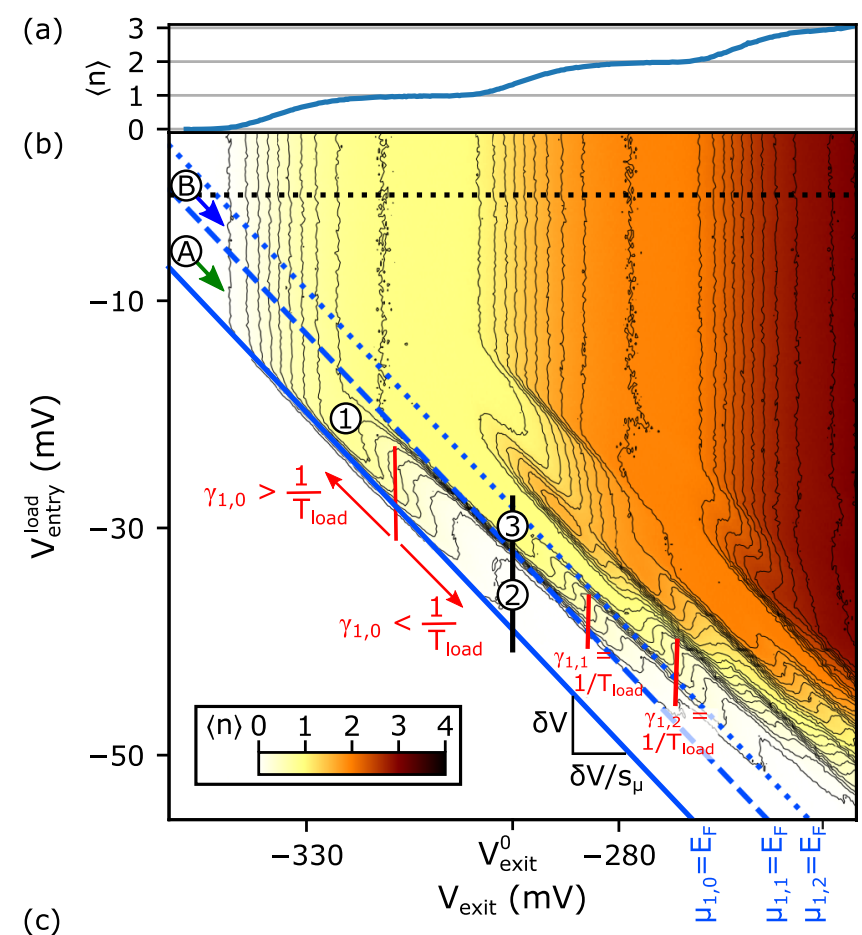

(c)

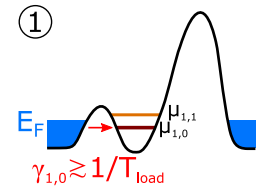

(2)

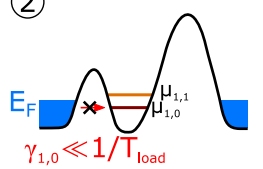

(3)

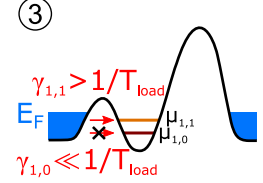

FIG. 2. (a) Quantized current steps are measured in the backtunneling-limited region [along the black dotted line in (b)]. (b) Measurement of the average number of captured electrons for $T_{\text {load }}=50 \mathrm{~ns}$. (c) Sketches of potential landscape during the loading phase (i), showing only the lowest two states. (1): Only the lowest SE state $\mu_{1,0}$ is below $E_{\mathrm{F}}$ and can be loaded. (2): The tunneling rate $\gamma_{1,0}$ is insufficient. (3): Loading to the second state $\mu_{1,1}$ dominates.

Hence the current at a given point $\left(V_{\text {entry }}^{\text {load }}, V_{\text {exit }}\right)$ near the lowerleft corner of the $\langle n\rangle=1$ plateau can be modeled by a product of two probabilities, $P_{\text {load }}$ for loading and $1-P_{\text {back }}$ for not backtunneling, respectively:

$$
\langle n\rangle=P_{\text {load }}\left(1-P_{\text {back }}\right) .
$$

Extracting $P_{\text {load }}=1-\exp \left[-\Gamma_{\text {in }}\left(V_{\text {entry }}^{\text {load }}, V_{\text {exit }}\right) T_{\text {load }}\right]$ gives direct information on the total intunneling rate $\Gamma_{\text {in }}$ during the loading stage. The other probability, $P_{\text {back }}=1-$ $\exp \left\{-\int \Gamma_{\text {back }}\left[V_{\text {entry }}(t), V_{\text {exit }}\right] d t\right\}$, depends on the electron escape rate $\Gamma_{\text {back }}$ during the backtunneling phase (ii), and can be parametrized as $P_{\text {back }}=1-\exp \left(-e^{-\alpha V_{\text {exit }}+\delta_{\text {back }}}\right)$ [19-22]. Both rates are defined by the same tunnel barrier, hence we expect the same exponential parametric dependence on the gate voltages for the in- and backtunneling rates, $\Gamma_{\text {in }}, \Gamma_{\text {back }} \propto e^{-\alpha V_{\text {exit }}}$ [23]. This is valid along the lines of constant energy, identified by a common slope $s_{\mu}$ as $\mu_{n, i}\left(V_{\text {entry }}, V_{\text {exit }}\right)=-k_{\mu}\left(V_{\text {entry }}+\right.$ $\left.s_{\mu} V_{\text {exit }}\right)+$ const in Fig. 2(b) $\left(k_{\mu}\right.$ is the measure of the gate lever arm; $s_{\mu}>0$ ). For dc voltage shifts along these lines, the time evolution of the QD energies is not affected, and hence neither the number of discrete states competing for loading, nor the timing for the onset of backtunneling $[20,22]$ change. 
(a)
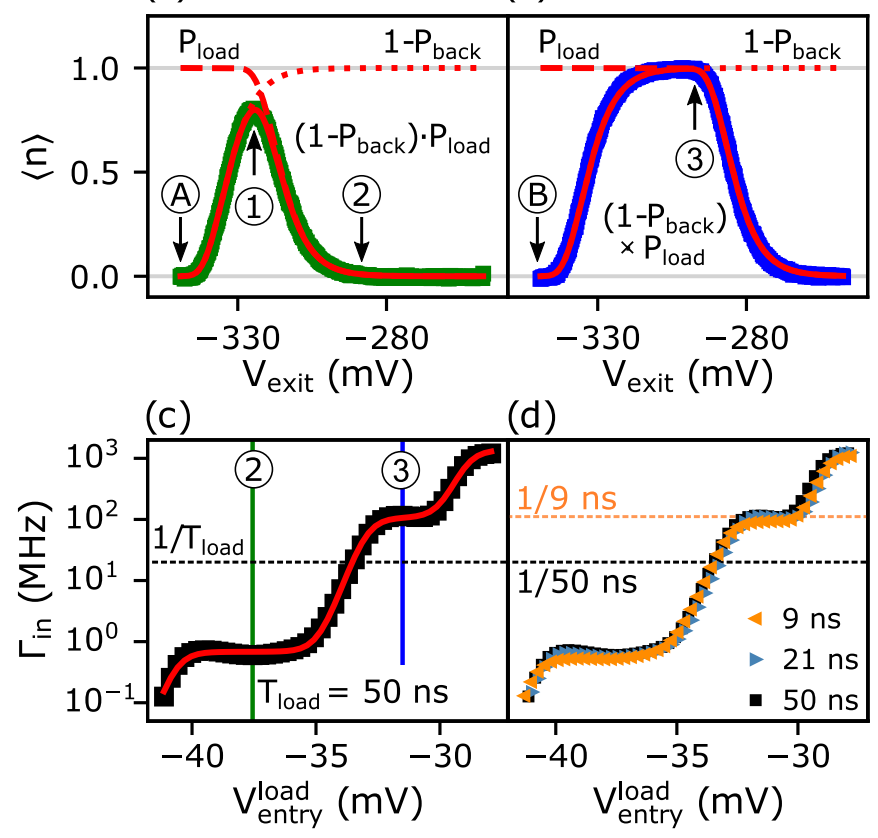

FIG. 3. (a),(b) Electron number $\langle n\rangle$ measured along a line of constant energy, starting at point (A) and point $(B)$ in Fig. 2(b), respectively. Red lines indicate fits using Eq. (1). (c),(d) Tunneling rates $\Gamma_{\text {in }}$ for fixed $V_{\text {exit }}=V_{\text {exit }}^{0}$ determined from fits for various values of $V_{\text {entry }}^{\text {load }}$ along the black line in Fig. 2(b) for (c) $T_{\text {load }}=9 \mathrm{~ns}$ and (d) $T_{\text {load }}=9,21$, and $50 \mathrm{~ns}$. Red line in (c) indicates a fit using Eq. (3).

Technically, we use the following ansatz to describe the gate voltage dependence of the intunneling rate:

$$
\Gamma_{\text {in }}\left(V_{\text {entry }}^{\text {load }}, V_{\text {exit }}\right)=W_{\text {in }}\left(V_{\text {entry }}^{\text {load }}+s_{\mu} \Delta V_{\text {exit }}\right) e^{-\alpha \Delta V_{\text {exit }}},
$$

where $\Delta V_{\text {exit }}=V_{\text {exit }}-V_{\text {exit }}^{0}$ is measured from an arbitrary chosen reference level $V_{\text {exit }}^{0}=-297 \mathrm{mV}$, and an unknown single-variable function $W_{\text {in }}$ describes the dependency of the rate on the depth of the QD during the loading stage. The function $W_{\text {in }}(V)$ is deduced by fitting Eq. (1) along the lines of constant $V=V_{\text {entry }}^{\text {load }}+s_{\mu} \Delta V_{\text {exit }}$ with $V$-independent globally optimized values of $s_{\mu}$ and $\alpha$ [24]. Two representative fits are shown in Figs. 3(a) and 3(b), differing only in the best-fit value of $W_{\text {in. }}$.

The method measures $\Gamma_{\text {in }}$ directly only when $\Gamma_{\text {in }}\left(V_{\text {entry }}^{\text {load }}, V_{\text {exit }}\right)$ is on the same order of magnitude as $1 / T_{\text {load }}$ [regions near the red lines $\gamma_{1, i}=1 / T_{\text {load }}$ in Fig. 2(b)], and relies on extrapolation of the exponential parametrization to extend the gate-voltage dependence via Eq. (2) to the whole SE loading region $\left(E_{\mathrm{F}}<\mu_{2,0}\right)$. The resulting $\Gamma_{\text {in }}\left(V_{\text {entry }}^{\text {load }}, V_{\text {exit }}^{0}\right)$ is shown in Fig. 3(c).

An important consistency check is independence of the inferred $\Gamma_{\text {in }}$ on the chosen value of $T_{\text {load }}$. Measurements with $T_{\text {load }}=9$ and $21 \mathrm{~ns}$ result in reduced loading probabilities compared to $T_{\text {load }}=50 \mathrm{~ns}$, but the extracted $\Gamma_{\text {in }}$ values agree well, as shown in Fig. 3(d).

Figures 3(c) and 3(d) show three clear steps of increased tunnel coupling corresponding to the (blue) constant-energy lines in Fig. 2(b). We parametrize the total rate by a discrete sum,

$$
W_{\text {in }}\left(V_{\text {entry }}^{\text {load }}\right)=\sum_{i=0}^{2} \gamma_{1, i}^{0} f\left(V_{\text {entry }}^{\text {load }}-V_{\text {entry }}^{1, i}\right),
$$

where $f(V)=\left[\exp \left(-k_{\mu} V / k_{\mathrm{B}} T_{\text {eff }}\right)+1\right]^{-1}$ is the Fermi distribution, $k_{\mathrm{B}}$ is the Boltzmann constant, and $V_{\text {entry }}^{1, i}$ is the value of $V_{\text {entry }}^{\text {load }}$ at which $\mu_{1, i}\left(V_{\text {entry }}^{\text {load }}, V_{\text {exit }}^{0}\right)=E_{\mathrm{F}}$. Equation (3) assumes that each state $\mu_{1, i}$ contributes to the total rate $\Gamma_{\text {in }}$ with $\gamma_{1, i}=$ $\gamma_{1, i}^{0} e^{-\alpha \Delta V_{\text {exit }}}$ when it is energetically possible according to the schematics of Fig. 2(c). Here $T_{\text {eff }}$ is the effective temperature and $T_{\text {eff }} / k_{\mu}, V_{\text {entry }}^{1, i}$, and $\gamma_{1, i}^{0}$ are used as fitting parameters [24]. In Fig. 3(c) the fit to Eq. (3) (red line) describes the data (squares) well. We find rather large differences of tunneling rates for consecutive SE states, $\gamma_{1,1} / \gamma_{1,0} \approx 160$ and $\gamma_{1,2} / \gamma_{1,1} \approx 12$.

In contrast to clear signatures of distinct SE states during the loading phase, the backtunneling probability, also inferred from fits to Eq. (1), shows no appreciable dependence on $V_{\text {entry }}^{\text {load }}$ This implies that by the end of loading the electron has relaxed to a state with a unique backtunneling rate, independent of the intunneling channel $i$. This is consistent with the expectation of strong relaxation from the excited orbital states $(i>0)$ to the SE ground state $(i=0)$ on a time scale that is much faster than the loading times of our experiment [25].

We further clarify the roles of relaxation by comparing several theoretical scenarios, all sharing the same gate-voltage dependence of individual tunneling rates inferred from the experiment via Eq. (3). In Fig. 4(a) we simulate the detector signal with the ground-state level $i=0$ only. Here, electrons capture succeeds only in a narrow parameter range, limited on the right by incomplete loading and on the left by backtunneling. In Fig. 4(b) we include three states, all quickly relaxing to $i=0$. The model accurately matches the experimental data in the relevant range [cf. Fig. 4(d)] as expected from the robustness of the fits in Fig. 3.

A hypothetical SE capture scenario in Fig. 4(c) simulates the same three states as in (b) but without internal relaxation [24]. The state-specific backtunneling rates $\gamma_{n, i}^{\mathrm{back}}$ are derived from $\gamma_{n, i}=g_{n, i} \gamma_{n, i}^{\text {back }}$ where $g_{n, i}$ is the degeneracy factor [22,23] ( $g_{1, i}=2$ for spin). In sharp contrast to (b), scenario (c) presents a gap in the current (region G1 in the figure) which can be seen to arise from a Coulomb blockade by a bettercoupled state [22]: for example, as soon as the excited SE state $(1,1)$ becomes energetically available at the start of the loading phase (i), it gets immediately occupied due to much larger tunnel coupling, thus blocking further loading into the ground state $(1,0)$ due to Coulomb repulsion. In this scenario without relaxation, the occupied $(1,1)$ state decays back to the source as soon as this becomes energetically possible in phase (ii), resulting in the gap G1.

Turning now to the experimental data on electron pairs, we note a similar gap [G2 in Fig. 4(d)] in the $n=2$ electron backtunneling line, shifting the latter from $V_{\text {exit }}=V_{\text {back }}^{2,0}$ toward more positive $V_{\text {exit }}=V_{\text {back }}^{2,1}$. This suggests initialization of an excited state $(2,1)$ which persists unrelaxed, and which has a backtunneling rate $\gamma_{2,1}^{\text {back }} / \gamma_{2,0}^{\text {back }} \approx \exp \left[\alpha\left(V_{\text {back }}^{2,1}-V_{\text {back }}^{2,0}\right)\right] \approx 5$ times higher than the ground state $(2,0)$. Extending the model to include loading and backtunneling of a second electron 


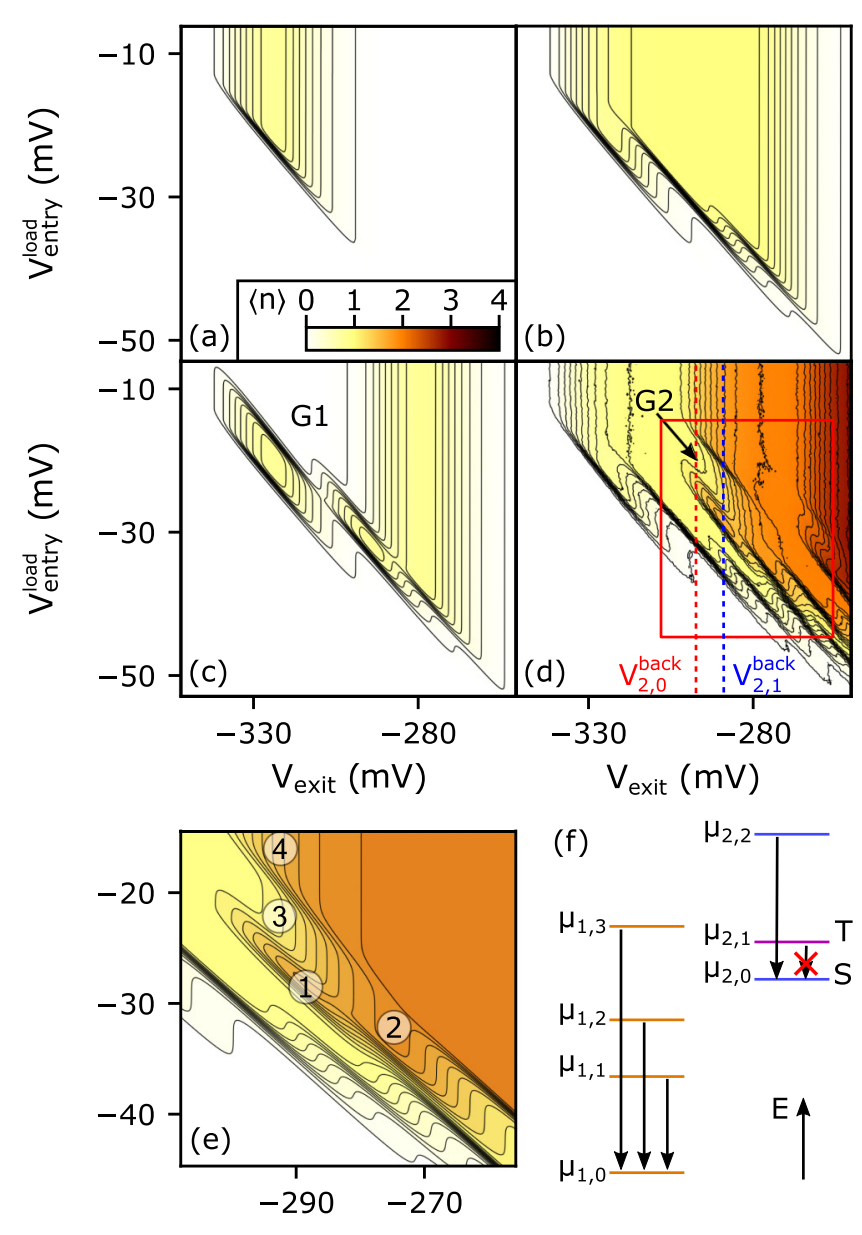

FIG. 4. (a)-(c) Simulations of the initialized electron number $\langle n\rangle$ for three models: (a) the SE ground state $i=0$ alone, (b) three SE states $(i=0,1,2)$ with immediate relaxation, and (c) three SE states $(i=0,1,2)$ without relaxation. (d) Measured $\langle n\rangle$ for the same gate-voltage range. (e) Simulation with multiple single- and twoelectron states with energies and relaxation paths depicted in (f). The corresponding region is highlighted by a red box in (d).

at discrete energies $\mu_{2, i}$ with appropriately chosen rates $\gamma_{2, i}$ and blocked $(2,1) \rightarrow(2,0)$ relaxation enables the simulation [Fig. 4(e)] to match quite well the structure of the observed energy-, intunneling-, and backtunneling-limited lines [cf. Fig. 4(d)]. The relative energies and relaxation paths assigned to the simulation are shown in Fig. 4(f), and quantitative details are given in the Supplemental Material [24].

The observed suppression of relaxation can be explained naturally by identifying $(2,0)$ and $(2,1)$ with singlet $(\mathrm{S})$ and triplet $(\mathrm{T})$ states, respectively, because triplet-to-singlet relaxation requires a spin flip [26] which occurs on a timescale typically much longer than our range of $T_{\text {load }}$ [27-29].

The key regions demonstrating control of electron pair initialization are marked in Fig. 4(e) as (1)-(4). (1): Only ground-state $S$ initialization is energetically allowed. (2) and (3): Loading into both $\mathrm{T}$ and $\mathrm{S}$ is allowed energetically but $\mathrm{T}$ is dynamically preferred due to a larger tunneling rate. The selectively initialized T state is kept at (2) but lost at (3) due to backtunneling. (4): Higher-energy S-type states enter, resulting in predominantly ground-state $S$ initialization via quick orbital relaxation paths not requiring a spin flip.

Switching the dc voltages between points (1) and (2) allows generation of electron pairs with on-demand selection between $\mathrm{S}$ and $\mathrm{T}$ states. The probability of capturing a pair instead of a SE can be estimated as $\langle n\rangle-1$. The corresponding maximal values in this demonstration for $T_{\text {load }}=50 \mathrm{~ns}$ are $55 \%$ and $80 \%$, for on-demand $\mathrm{S}$ and $\mathrm{T}$, respectively; both values are straightforward to improve by increasing $T_{\text {load}}$. Furthermore, remaining SE charge states could easily be distinguished and rejected in applications. The fidelity of preparation for any of the three degenerate components $T_{0}$, $T_{-}, T_{+}\left(g_{2,1}=3\right)$ of the excited state $\mathrm{T}$ versus the ground state $\mathrm{S}\left(g_{2,0}=1\right)$ at (2) can be approximated by the ratio of the corresponding intunneling rates, $\gamma_{2,1} / \gamma_{2,0} \approx 15$. These values are dictated by the energy gap and the barrier selectivity inherent to a particular device; we envision the fidelity can be further improved by optimizing the confinement potential of the QD.

In summary, we have presented a method to identify, tune, and measure tuneling rates for discrete single- and twoelectron quantum states of a semiconductor QD. Exponential energy dependence of the individual rates enable selective initialization of quantum states on the timescales shorter than spin-relaxation time. Our device can be used as an electron "entangler" for on-demand emission of electron pairs with deterministically controlled exchange symmetry for applications in electron quantum optics $[9,10,30]$ and quantum information transfer $[13,14]$.

This work was supported in part by the Joint Research Projects e-SI-Amp (15SIB08) and SEQUOIA (17FUN04). These projects received funding from the European Metrology Programme for Innovation and Research (EMPIR) cofinanced by the Participating States and from the European Unions Horizon 2020 research and innovation programme. J.K. has been supported by University of Latvia Grant No. AAP2016/B031. We thank Holger Marx and Klaus Pierz for MBE growth, Thomas Weimann for electron-beam lithography, and Rolf J. Haug for stimulating discussions.
[1] D. P. DiVincenzo, The physical implementation of quantum computation, Fortschr. Phys. 48, 771 (2000).

[2] E. Knill, Quantum computing with realistically noisy devices, Nature (London) 434, 39 (2005).

[3] D. Loss and D. P. DiVincenzo, Quantum computation with quantum dots, Phys. Rev. A 57, 120 (1998).
[4] J. R. Petta, A. C. Johnson, J. M. Taylor, E. A. Laird, A. Yacoby, M. D. Lukin, C. M. Marcus, M. P. Hanson, and A. C. Gossard, Coherent manipulation of coupled electron spins in semiconductor quantum dots, Science 309, 2180 (2005).

[5] F. H. L. Koppens, C. Buizert, K. J. Tielrooij, I. T. Vink, K. C. Nowack, T. Meunier, L. P. Kouwenhoven, and L. M. K. 
Vandersypen, Driven coherent oscillations of a single electron spin in a quantum dot, Nature (London) 442, 766 (2006).

[6] F. Stein, H. Scherer, T. Gerster, R. Behr, M. Götz, E. Pesel, C. Leicht, N. Ubbelohde, T. Weimann, K. Pierz, H. W. Schumacher, and F. Hohls, Robustness of single-electron pumps at sub-ppm current accuracy level, Metrologia 54, S1 (2017).

[7] B. Kaestner and V. Kashcheyevs, Non-adiabatic quantized charge pumping with tunable-barrier quantum dots: A review of current progress, Rep. Prog. Phys. 78, 103901 (2015).

[8] E. Bocquillon, F. D. Parmentier, C. Grenier, J.-M. Berroir, P. Degiovanni, D. C. Glattli, B. Plaçais, A. Cavanna, Y. Jin, and G. Fève, Electron Quantum Optics: Partitioning Electrons One by One, Phys. Rev. Lett. 108, 196803 (2012).

[9] N. Ubbelohde, F. Hohls, V. Kashcheyevs, T. Wagner, L. Fricke, B. Kästner, K. Pierz, H. W. Schumacher, and R. J. Haug, Partitioning of on-demand electron pairs, Nat. Nanotechnol. 10, 46 (2015).

[10] C. Bäuerle, D. C. Glattli, T. Meunier, F. Portier, P. Roche, P. Roulleau, S. Takada, and X. Waintal, Coherent control of single electrons: A review of current progress, Rep. Prog. Phys. 81, 056503 (2018).

[11] M. Yamamoto, S. Takada, C. Bäuerle, K. Watanabe, A. D. Wieck, and S. Tarucha, Electrical control of a solid-state flying qubit, Nat. Nanotechnol. 7, 247 (2012).

[12] T. A. Baart, M. Shafiei, T. Fujita, C. Reichl, W. Wegscheider, and L. M. K. Vandersypen, Single-spin CCD, Nat. Nanotechnol. 11, 330 (2016).

[13] T. Fujita, T. A. Baart, C. Reichl, W. Wegscheider, and L. M. K. Vandersypen, Coherent shuttle of electron-spin states, npj Quantum Inf. 3, 22 (2017).

[14] H. Flentje, P.-A. Mortemousque, R. Thalineau, A. Ludwig, A. D. Wieck, C. Bäuerle, and T. Meunier, Coherent longdistance displacement of individual electron spins, Nat. Commun. 8, 501 (2017).

[15] C. Leicht, B. Kaestner, V. Kashcheyevs, P. Mirovsky, T. Weimann, K. Pierz, and H. W. Schumacher, Non-adiabatic pumping of single electrons affected by magnetic fields, Phys. E (Amsterdam, Neth.) 42, 911 (2010), 18th International Conference on Electron Properties of Two-Dimensional Systems.

[16] S. Amasha, K. MacLean, I. P. Radu, D. M. Zumbühl, M. A. Kastner, M. P. Hanson, and A. C. Gossard, Spin-dependent tunneling of single electrons into an empty quantum dot, Phys. Rev. B 78, 041306(R) (2008).

[17] F. Stein, D. Drung, L. Fricke, H. Scherer, F. Hohls, C. Leicht, M. Götz, C. Krause, R. Behr, E. Pesel, K. Pierz, U. Siegner, F. J. Ahlers, and H. W. Schumacher, Validation of a quantizedcurrent source with $0.2 \mathrm{ppm}$ uncertainty, Appl. Phys. Lett. 107, 103501 (2015).
[18] M. Pioro-Ladrière, J. H. Davies, A. R. Long, A. S. Sachrajda, L. Gaudreau, P. Zawadzki, J. Lapointe, J. Gupta, Z. Wasilewski, and S. Studenikin, Origin of switching noise in $\mathrm{GaAs} / \mathrm{Al}_{x} \mathrm{Ga}_{1-x}$ As lateral gated devices, Phys. Rev. B 72, 115331 (2005).

[19] V. Kashcheyevs and B. Kaestner, Universal Decay Cascade Model for Dynamic Quantum Dot Initialization, Phys. Rev. Lett. 104, 186805 (2010).

[20] L. Fricke, M. Wulf, B. Kaestner, V. Kashcheyevs, J. Timoshenko, P. Nazarov, F. Hohls, P. Mirovsky, B. Mackrodt, R. Dolata, T. Weimann, K. Pierz, and H. W. Schumacher, Counting Statistics for Electron Capture in a Dynamic Quantum Dot, Phys. Rev. Lett. 110, 126803 (2013).

[21] G. Yamahata, K. Nishiguchi, and A. Fujiwara, Accuracy evaluation and mechanism crossover of single-electron transfer in $\mathrm{Si}$ tunable-barrier turnstiles, Phys. Rev. B 89, 165302 (2014).

[22] A. Rossi, J. Klochan, J. Timoshenko, F. E. Hudson, M. Möttönen, S. Rogge, A. S. Dzurak, V. Kashcheyevs, and G. C. Tettamanzi, Gigahertz single-electron pumping mediated by parasitic states, Nano Lett. 18, 4141 (2018).

[23] K. MacLean, S. Amasha, I. P. Radu, D. M. Zumbühl, M. A. Kastner, M. P. Hanson, and A. C. Gossard, Energy-Dependent Tunneling in a Quantum Dot, Phys. Rev. Lett. 98, 036802 (2007).

[24] See Supplemental Material at http://link.aps.org/supplemental/ 10.1103/PhysRevB.99.201409 for details on experimental setup, fitting and simulation procedures, and additional measurements.

[25] P. Stano and J. Fabian, Orbital and spin relaxation in single and coupled quantum dots, Phys. Rev. B 74, 045320 (2006).

[26] R. Hanson, L. P. Kouwenhoven, J. R. Petta, S. Tarucha, and L. M. K. Vandersypen, Spins in few-electron quantum dots, Rev. Mod. Phys. 79, 1217 (2007).

[27] T. Fujisawa, D. G. Austing, Y. Tokura, Y. Hirayama, and S. Tarucha, Allowed and forbidden transitions in artificial hydrogen and helium atoms, Nature (London) 419, 278 (2002).

[28] S. Sasaki, T. Fujisawa, T. Hayashi, and Y. Hirayama, Electrical Pump-and-Probe Study of Spin Singlet-Triplet Relaxation in a Quantum Dot, Phys. Rev. Lett. 95, 056803 (2005).

[29] R. Hanson, L. H. Willems van Beveren, I. T. Vink, J. M. Elzerman, W. J. M. Naber, F. H. L. Koppens, L. P. Kouwenhoven, and L. M. K. Vandersypen, Single-Shot Readout of Electron Spin States in a Quantum Dot Using Spin-Dependent Tunnel Rates, Phys. Rev. Lett. 94, 196802 (2005).

[30] J. D. Fletcher, P. See, H. Howe, M. Pepper, S. P. Giblin, J. P. Griffiths, G. A. C. Jones, I. Farrer, D. A. Ritchie, T. J. B. M. Janssen, and M. Kataoka, Clock-Controlled Emission of SingleElectron Wave Packets in a Solid-State Circuit, Phys. Rev. Lett. 111, 216807 (2013). 